\title{
Toward a Trans-textual, Interdisciplinary, Cross- cultural, and Transmodern Community
}

English version

Tonglin Lu

\section{(2) OpenEdition}

\section{Journals}

Electronic version

URL: http://journals.openedition.org/transtexts/173

DOI: 10.4000/transtexts. 173

ISSN: 2105-2549

\section{Publisher}

Gregory B. Lee

Printed version

Date of publication: 1 May 2006

Number of pages: $44-45$

ISSN: 1771-2084

\section{Electronic reference}

Tonglin Lu, «Toward a Trans-textual, Interdisciplinary, Cross-cultural, and Transmodern Community », Transtext(e)s Transcultures 跨文本跨文化 [Online], 1 | 2006, Online since 13 September 2009, connection on 04 May 2019. URL : http://journals.openedition.org/transtexts/173 ; DOI : 10.4000/ transtexts. 173 


\title{
Toward a Trans-textual, Interdisciplinary, Cross-cultural, and Transmodern Community BY Lu Tonglin
}

\begin{abstract}
The current globalization process, intensified by the end of the cold war, has changed the geopolitical map. We no longer live in a world centered on the West as the reference according to which the rest of the world measured its modernity and progress, but in a trans-modern world (if we borrow the expression from Enrique Dussel) where different cultures links with each other through transnational ties. ${ }^{1}$ Does this trans-modernity mean that the value of the West has been universalized through the expansion of global capitalism —as suggested by Fredric Jameson? ? Or has the trans-modernity gradually pushed the pattern of single culture dominance to the background?

The contemporary world has changed so quickly and often, so chaotically that it is difficult, if not impossible, to give a black and white answer to these questions. The cultural change brought about by the intensified process of globalization, however, is more visible outside the previous center, the West. Modernization in a non-Western culture was often considered tantamount to Westernization, namely, remaking its own culture in the image of Europe, which represented progress, rationality, and modernity. This was the case in China. Since the May Fourth movement at the beginning of the twentieth century, political parties and schools of thought in China have found reference points in the West across a wide ideological spectrum, from nationalism to communism. In this sense, we can say that modernity is measured not only by chronological development from past to present, but also by spatial distance from peripheries to the center. Thus, the modern age has also created the image of Europe as the center of the world, or the center of reference of "universal" values.

By contrast, led by multinational corporations and relatively free from the restrictions of national boundaries, the current process of intensified globalization has a decentralizing effect as capital flows toward maximum profits. In this context, decentralization does not imply democracy, but merely indicates the absence of a moral center as the ultimate reference. Although this decentralization is designed to benefit the financial power of multinational corporations, the amorphous nature of this power has also gradually freed the collective imaginary in the developing world from the cultural complex created by the myth of modernity, Eurocentrism. As a result, instead of looking for salvation in the implicit or explicit center of ultimate reference, the previously marginalized world is in the process of developing multi-centers outside the West, as in the case of the Asian-pacific area.

In other words, transnational ties have gradually replaced the binary division between the West and the rest. This change has been translated in new intellectual vision especially outside the West. For example, Traces, another multilingual journal of Translation and Cultural Theory, has been published in English, Chinese, Japanese and Korean. This journal emphasizes
\end{abstract}

1 See Enrique Dussel, "Beyond Eurocentrism: the World-System and the Limits of Modernity," in Fredric Jameson and Masao Myoshi (eds.), The Cultures of Globalization, Durham, Duke University Press, 1998, pp. 3-31. 2 Fredric Jameson, A Singular Modernity, London, Verso, 2002, p. 12. 
transnational ties among Northeast Asian cultures. The perception of the world of the binary opposition between the West and the rest, however, has still been deeply rooted in Western academy. Because of its institutional settings, and disciplinary divisions, as its members we are often slow to respond to the geopolitical changes occurred in recent years.

Theorists like Jameson still talk about a singular modernity, modernity of Western capitalism, while scholars in postcolonial studies emphasize the victimization of non-Western cultures by the center. For Jameson, because capitalism originated in Anglo-Saxon society, any expansion means further universalizing its culture. But if not a single society monopolized feudalism, why should capitalism carry such a specific cultural trademark ? Does this suggest that the centralized position of Western culture remains eternal despite any change occurred in global culture? Further, what is now perceived as Western culture, or more specifically Anglo-Saxon culture, can be traced back to historical influences of multiple non-western origins. Why should modernity exclusively be marked by one culture, while not a single culture can claim the same exclusiveness?

It is easier to stick to the pattern of victimization to explain problems existing in cultures generally considered peripheral in the past. The question is: do so-called non-western cultures remain as voiceless in today's increasingly globalized world as in their colonial past? Can we measure various cultures in terms of one single center disregarding transnational ties among them ? By doing so, do we contribute to reinforcing its image of the center by referring it as such even through negations? Further, does our effort to emphasize the powerlessness of these cultures serve more to empower our own voices than to understand these cultures in their historical and contemporary settings?

As a Chinese film scholar, I particularly feel that this mode of thinking has become more and more inadequate in a decentralized global village. For example, Asian Cinema has recently achieved a high profile in international film culture. Despite its orientation toward transnational and global film production, however, scholars in Asian cinema especially in North America, still remains relatively separated in our research areas, roughly confined within the national (or regional, in the case of sinophone film for example) boundaries. In other words, limited by the notion of national cinema and the binary division between the West and the rest, Asian cinema as a scholarly field in Western academy is still defined mainly as the other of, or difference from, its Western counterpart.

The intensified process of globalization has inadvertently broken away from the pattern of single culture dominance mainly in order to facilitate the expansion of global capital. It is no longer possible to resist global capitalism by sticking to the old world map centered on a traditional imperialist center. At the same time, a multicultural discourse risking uncritically overlapping with the language of multinational corporations, thus, it may be absorbed in the system of global capitalism. Stuart Hall describes cultural difference as ambiguous and cultural identity as becoming. ${ }^{3}$ How can our journal contribute to the new becoming of a transnational, trans-textual, cross-culture, interdisciplinary, and transmodern intellectual community, to form a culture of resistance? 


\section{Vers une communauté trans-textuelle, interdisciplinaire, transculturelle et transmoderne}

Le processus actuel de mondialisation, intensifié par la fin de la Guerre Froide, a changé la carte géopolitique mondiale. Nous ne vivons plus dans un monde où l'Occident est le point de référence selon lequel le monde mesurait modernité et progrès, mais plutôt dans un monde transmoderne (pour employer l'expression d'Enrique Dussel) où différentes cultures se connectent au moyen de liens transnationaux. ${ }^{4}$ Cette transmodernité signifie-t-elle que la valeur de l'Occident a été universalisée à travers l'expansion du capitalisme global - comme le suggère Fredric Jameson. ${ }^{5}$ Ou la transmodernité a-t-elle repoussé à l'arrière-plan le modèle de domination d'une seule culture?

Le monde contemporain a changé si rapidement et si fréquemment, de manière si troublée qu'il est difficile, sinon impossible, d'apporter une réponse catégorique à ces questions. Cela étant, le changement culturel engendré par le processus intensifié de la mondialisation est plus visible en-dehors du centre précédent, à savoir l'Occident. La modernisation dans une culture non-occidentale était souvent considérée comme une occidentalisation, c'est-à-dire un refaçonnement de sa propre culture à l'image de l'Europe, qui représentait le progrès, la rationalité et la modernité . Ce fut le cas en Chine. Depuis le Mouvement du 4 Mai au début du vingtième siècle, les partis politiques et les écoles de pensée en Chine sont allés chercher leurs points de référence en Occident, dans un large éventail idéologique allant du nationalisme au communisme. De cette manière, on peut dire que la modernité se mesure non seulement suivant le développement chronologique du passé au présent, mais aussi selon la distance spatiale de la périphérie vers le centre. Ainsi, l'âge moderne a aussi créé l'image de l'Europe en tant que centre du monde, ou en tant que centre de référence des valeurs « universelles».

En contraste, le processus actuel de mondialisation intensifiée, avec à sa tête des corporations multinationales et relativement libéré des restrictions posées par les frontières nationales, ce processus a un effet décentralisateur alors que le capital grimpe vers des profits maximum. Dans ce contexte, la mondialisation ne signifie pas démocratie, mais indique seulement l'absence d'un centre moral comme ultime référence. Bien que cette décentralisation ait pour but de profiter au pouvoir financier des corporations multinationales, la nature amorphe de ce pouvoir a en même temps progressivement libéré l'imaginaire collectif du monde en voie de développement du complexe d'infériorité culturelle créé par le mythe de la modernité, l'Eurocentrisme. Le résultat c'est qu'au lieu de rechercher le salut dans le centre implicite ou explicite de référence ultime, le monde marginalisé dans le passé est en train de développer des multi-centres en dehors de l'Occident, comme c'est le cas dans la zone Asie-Pacifique. En d'autres termes, les liens transnationaux ont peu à peu remplacé la division binaire entre l'Occident et le reste du monde. Ce changement s'est traduit par une nouvelle vision intellectuelle en particulier en dehors de l'Occident. Par exemple, Traces, un autre journal multilingue de traduction et théorie de la culture a été publié en anglais, chinois, japonais et

4 Enrique Dussel, "Beyond Eurocentrism: the World-System and the Limits of Modernity" in Friedric Jameson et Masao Myoshi (eds.), The Cultures of Globalization, Durham, Duke University Press, 1998. pp. 3-31. 5 Fredric Jameson, A Singular Modernity, London, Verso, 2002. p. 12. 
coréen. Ce journal met l'accent sur les liens transnationaux entre les cultures de l'Asie du Nord. La perception du monde d'opposition binaire entre l'Occident et le reste du monde est toujours bien ancrée cependant dans le monde universitaire occidental. En raison de ses institutions et de ses divisions disciplinaires, nous, en tant que membres de ce monde, nous répondons souvent tardivement aux changements géopolitiques de ces dernières années.

Les théoriciens comme Jameson parlent toujours d'une modernité au singulier, celle du capitalisme occidental, alors que les chercheurs en études post-coloniales mettent l'accent sur la victimisation des cultures non-occidentales par le centre. Pour Jameson, comme le capitalisme est issu d'une société anglo-saxonne, toute expansion de ce système signifie l'universalisation de sa culture. Mais si le féodalisme n'était pas l'apanage d'une seule société pourquoi en irait-il différemment du capitalisme ? Cela signifie-t-il que la position centralisée de l'Occident demeure éternelle en dépit de tous les changements intervenant dans la culture mondiale ? De plus, ce que l'on entend à présent par culture occidentale, ou plus spécifiquement culture anglo-saxonne, a subi les influences historiques de nombreuses origines non-occidentales. Pourquoi la modernité devrait-elle être exclusivement marquée par une seule culture, alors que nulle culture ne peut revendiquer la même exclusivité ?

II est plus aisé de s'en tenir au modèle de la victimisation pour expliquer les problèmes existant dans les cultures considérées dans le passé comme secondaires. Le problème est de savoir si les cultures prétendues non-occidentales sont tout aussi muettes dans le monde toujours plus mondialisé d'aujourd'hui qu'elles ne l'étaient dans leur passé colonial. Pouvonsnous évaluer les cultures à l'aune d'un seul centre sans égard aux liens transnationaux qui les unissent? Et ce faisant, ne contribuons-nous pas à renforcer l'image du centre en y faisant référence comme tel même à travers les négations ? De plus, notre effort de mise en relief de l'impuissance de ces cultures sert-il plus à donner de la puissance à nos propres voix plutôt qu'à comprendre ces cultures dans leurs cadres historiques et contemporains ?

En tant que chercheuse chinoise du cinéma, je ressens tout particulièrement l'insuffisance de ce mode de pensée dans le village global décentralisé. Ainsi, le cinéma asiatique a-t-il acquis récemment ses lettres de noblesse dans la culture filmique internationale. Cependant, en dépit de son orientation vers une production filmique transnationale et globale, les chercheurs en cinéma asiatique, particulièrement en Amérique du Nord, restent cloisonnés dans leurs domaines de recherche respectifs, à l'intérieur de frontières nationales (ou régionales comme c'est le cas du film sinophone par exemple). En d'autres termes, limité par la notion du cinéma national et par la division entre l'Occident et le reste du monde, le cinéma asiatique en tant que champ d'étude des universités occidentales se définit toujours comme l'autre, ou la différence de son équivalent occidental.

Le processus intensifié de la mondialisation a, par inadvertance, rompu d'avec le modèle de la domination d'une seule culture afin de faciliter l'expansion du capital mondial. II n'est plus possible de résister au capitalisme mondial en s'en tenant à la vieille carte du monde avec son centre impérialiste traditionnel. En même temps, un discours multiculturel risque de coïncider avec celui des corporations multinationales et ainsi risque d'être absorbé dans le système du capitalisme global. Stuart Hall disait que la différence culturelle était ambiguë là où l'identité culturelle était devenir. ${ }^{6}$ Comment notre journal peut-il contribuer au devenir d'une communauté transnationale, trans-textuelle, transculturelle, interdisciplinaire et transmoderne afin de former une culture de résistance?

6 Stuart Hall, « Who Needs Identity ? » in Stuart Hall et Paul du Gay (eds.), Questions of Cultural Identity, London, Sage, 1996. pp. 1-17. 


\section{迈向一个跨文本、 \\ 跨学科、跨文化和跨现代的共同体}

加速于冷战期间的现有全球化进程已经改变了地缘政治的地图。我们不再生存 在一个以“西方” 为中心，也就是以西方来衡量其它的世界现代性和发展进 程的世界中。借用杜塞尔 (Enrique Dussel) 的说法, 我们现在生存在一个 “跨 现代”的世界中。7 在这样一个跨现代的世界中，不同的文化由跨国家的关系 而彼此联结。这种 “跨现代性” 是否正如詹明信 (Fredric Jameson) 所指出的那 样，表示 “西方” 的价值已经被全球性的资本主义扩张所普世化? ${ }^{8}$ 还是说这 种“跨现代性”已经逐渐将单一文化统治的模式推向后台?

近代世界变化如此急剧频密, 混乱不堪, 我们难以甚至无法对以上这些问题给 出一个黑白分明的答案。由激化的全球化进程所带来的文化变异在过去的 “西 方” 中心之外更为显著。在一种“非西方” 的文化中，现代化往往被认为是西 方化的同义词。这也就是说，现代化按照代表着进步、理性和现代性的欧洲形 象重塑着原有的文化。中国的情况正是如此。自二十世纪初的五四运动始，中 国的政党和思想流派在西方广阔的思想范围中，从民族主义以至共产主义，找 到了各自的参照之处。在这种意义上，我们可以说现代化不仅仅以一个从古至 今的时间次序衡量，同时也是以边缘到中心的空间范围来衡量的。因此，现代 历史也同时创造了这样的欧洲形象——世界中心，或 “普世”价值的参照中 心。

与之相反, 现今这种被激化的全球化进程受控于跨国集团, 相对来说未受制于 国家边境之限, 却于资本涌向最大利益之际产生了一种非中心化的后果。在这 种情境之中, 非中心化并不含有民主之义, 却仅仅表明着道德中心作为终极参 照物的缺场。虽然该非中心化原本是为了使跨国集团的经济权力受益, 但此经 济权力无形无象的特性却同时也使发展中世界的集体幻想得以抽离于现代性迷 思所创造的“欧洲中心主义” 文化情结。其结果是, 曾被边缘化的世界不再从 （欧洲这个）作为终极参照物的隐形或显性中心寻找救赎, 而致力于在西方以 外的世界发展多元中心。如亚太地区就是如此。

换言之, 种种跨国联系已逐渐取代了西方与非西方之间的二元分界。此种改变 已被诠释于一种全新的智性视野中，在西方之外尤为如此。譬如说，另一本关 于翻译与文化理论的多语杂志《Traces》已以英文、中文、日文及韩文出版。 该杂志对东北亚各文化之间的跨国联系尤为关注。当然, 西方和非西方之间二 元分界的世界观仍深深植根于西方学界中。我们身在此中, 深受西方学界的组 织架构和学科分野影响, 因而对近年来的地缘政治变化反映颇为迟钝。

以詹明信(Jameson)为例的理论家仍在高谈单一的现代性, 西方资本主义的现代 性, 与此同时, 后殖民主义研究者却强调着非西方文化成为西方中心牺牲品 的这样一个过程。对于詹明信而言, 由于资本主义源于盎格鲁-撒克逊（Anglo-

7 Enrique Dussel, "Beyond Eurocentrism: the World-System and the Limits of Modernity" in Friedric Jameson et Masao Myoshi (eds.), The Cultures of Globalization, Durham, Duke University Press, 1998. pp. 3-31, 8 Fredric Jameson, A Singular Modernity, London, Verso, 2002. p. 12. 
Saxon）社会，资本主义的任何扩张都意味着其源文化的普世化。问题是，既 然封建主义并非为单一社会所垄断，为何资本主义会带有这一特有的文化印 记? 这是否表明即使全球文化进程发生任何变化，西方文化的中心化地位都永 固不变? 再进一步说，人们所理解的 “西方文化”，或在特指意义上说，盎格 鲁-撒克逊文化，可以追溯到多个非西方起源的历史影响上。既然没有任何一 种文化能够自称拥有同样的排他性，现代性又如何可能仅仅由一种文化而代 表?

坚持此种 “被牺牲品化” 的思维模式更易于解释那些曾经被认为是边缘文化中 存在的问题。问题在于，在目前更为全球化的世界中，那些所谓的“非西方文 化” 仍然象在它们的殖民历史上那样保持沉默吗? 我们能否用单一中心来衡量 多样的文化而无视于它们之间的跨国联系? 如果真的这样做的话, 我们在如此 甚至完全提及其对立面的同时，是否反而强化了它作为中心的形象呢? 再进一 步说，我们在致力于强调其他非西方文化的无力状态的同时，是否反而强化了 我们自身的声音，而无助于在这些文化的过去和当代背景下对它们进行理解 呢?

作为一名中国电影学者，我深感以上这种二元思维模式在一个非中心化的全球 村中已经愈发稀缺。譬如说, 亚洲电影近来在国际电影文化中获得了颇高声 誉。然而, 尽管亚洲电影已步向跨国境和全球化电影制作大局, 研究亚洲电影 的学者们, 尤其是那些北美的学者们, 仍然在我们这个研究领域中保持着一定 的游离性。他们把自己限制在国家范畴或者地区范畴（在华语电影研究中便 是如此）之内。换言之，由于受到国家性电影和西方/非西方的二元化分野理 念的限制，作为西方学术界中一个研究领域的亚洲电影仍被大致定义为其 “西 方” 同类的 “他者” 或相异的形式。

全球化的加速进程已经无可逆转地从单一文化主宰的模式中挣脱出来，而这主 要出于使全球资本扩张成为可能的目的。以坚守那幅围绕于传统的帝国主义中 心的过时世界地图而抗拒全球资本主义的做法已经不复可行。与此同时，一种 多元文化的话语正在尝试不带批判性地向跨国集团的语言靠拢。由此，这种 话语将得以被全球资本主义体系所吸纳。在斯图亚特. 霍尔(Stuart Hall)的描述 中, 文化差异模糊而抽象, 文化身份是一种生成过程。9 我们这本杂志如何才 能为这种跨国、跨文本、跨文化、跨学科和跨现代的知识界共同体的生成过程 尽一份力，从而营造一种具有抵抗力的文化?

9 Stuart Hall, « Who Needs Identity ? » in Stuart Hall et Paul du Gay (eds.), Questions of Cultural Identity, London, Sage, 1996, pp. 1-17. 